\title{
Diferentes níveis de gordura na dieta de vacas Jersey em lactação influenciam a resposta superovulatória?
}

\author{
Dietary fat levels to lactating Jersey cows can influence superovulatory response?
}

\author{
Anelis Cristina Coscioni ${ }^{5}$ Lígia Margareth Cantarelli Pegoraro ${ }^{1}$ \\ Cláudio Alves Pimentel ${ }^{3}$ Vivian Fischer ${ }^{4}$ José Eduardo Portela Santos $^{2}$ \\ Waldyr Stumpf Júnior ${ }^{1}$
}

\section{RESUMO}

O objetivo deste trabalho foi avaliar diferentes níveis de gordura na dieta de vacas em lactação, sobre a resposta superovulatória e qualidade embrionária. Foram utilizadas dezoito vacas Jersey $(420 \pm 70,6 \mathrm{~kg}$ de peso vivo; $22,2 \pm 1,7 \mathrm{~kg}$ de leite) agrupadas em blocos de acordo com a data de parição e ordem de lactação e aleatoriamente distribuídas nos tratamentos. As dietas consistiram de: controle- dieta a base de milho e farelo de soja contendo 4,0\% de extrato etéreo (EE); médio- dieta controle adicionada de sebo bovino para aumentar o extrato etéreo da dieta para $6,0 \%$ e alto- dieta controle adicionada de sebo bovino para se obter extrato etéreo de $8,0 \%$ na dieta. Todos os animais foram superovulados duas vezes (aos 90 e 130 dias pósparto), sendo as coletas realizadas sete dias após a inseminação artificial (IA). As estruturas coletadas foram avaliadas para estágio de desenvolvimento e qualidade embrionária (IETS, 1999). Não foram encontradas diferenças significativas com relação ao número de estruturas coletadas, corpos lúteos, embriões de grau um e dois, e embriões de grau quatro. O grupo que recebeu alta $(2,9 \pm 0,4)$ gordura apresentou maior número de embriões de grau três em relação aos grupos controle $(0,2 \pm 0,4)$ e médio $(0,0 \pm 0,5)$. A adição de sebo para aumentar o EE em dietas vacas lactantes não melhorou a resposta superovulatória e a qualidade embrionária.

Palavras-chave: níveis de gordura na dieta, vacas leiteiras, embriões, superovulação.

\begin{abstract}
This experiment aims at evaluating the effects of dietary fat to lactating dairy cows, on the superovulatory response and embryo quality. Eighteen high producing lactating Jersey cows $(420 \pm 70.6 \mathrm{~kg}$ of live weight, $22.2 \pm 1.7 \mathrm{~kg}$ of milk/day) were grouped according to calving date and lactation order, and randomly assigned to one of the three treatment diets. The control group received a basic diet with corn and soybean bran, containing $4.0 \%$ ether extract, the medium group received the control diet with additional tallow to increase the ether extract content to $6.0 \%$ and the high group treatment received the control diet with additional tallow to increase the ether extract content to $8.0 \%$. All cows were twice superovulated, at 90 and 130 days postpartum. The structures recovered (7 days after artificial insemination) were evaluated for developmental stage and quality grade (IETS, 1999). The results shows no difference on structures number, embryos grade one and two, grade four and corpora lutea number, for the tested treatments. The cows groups that received high fat diet $(2.9 \pm 0.4)$ had higher number of grade three embryos in relationship to control $(0.2 \pm 0.4)$ and medium $(0.0 \pm 0.5)$ groups. Addition of tallow to diets to increase EE levels to lactating cows did not increase the superovulatory response and embryo quality.
\end{abstract}

Key words: fat levels on diet, milk cows, embryo, superovulation.

${ }^{1}$ Pesquisador, Embrapa Clima Temperado, Pelotas, BR 392 km 78, 96001970, CP 403, Pelotas, RS, Brasil. E-mail: ligia@cpact.embrapa.br

${ }^{2}$ Professor, School of Veterinary Medicine, Davis, Califórnia.

${ }^{3}$ Professor, Faculdade de Veterinária, Universidade Federal de Pelotas (UFPel), Pelotas, RS, Brasil.

${ }^{4}$ Professor, Programa de Pós-graduação em Zootecnia, UFPel, Pelotas, RS, Brasil.

${ }^{5}$ Aluno do Doutorado PPGZ, UFPel, Pelotas, RS, Brasil. 


\section{INTRODUÇÃO}

A eficiência da produção de leite frente à adição de fontes de gordura à dieta de vacas de alta produção, tem sido enfoque de alguns trabalhos realizados na Embrapa - Clima Temperado (LÓPEZ et al., 2003; NÖRNBERG et al., 2004), que avaliaram os efeitos de fontes como o sebo bovino, gordura protegida e farelo de arroz integral. Seqüencialmente, foram avaliados os efeitos da adição de níveis de sebo sobre a produção de leite e a reprodução.

Nas últimas décadas, o melhoramento genético, através da seleção para características produtivas, gerou animais com grande capacidade de produção leiteira. WEIGEL (2001) destacou que a vaca média americana produz mais do que o dobro do que produzia há quarenta anos e isto se deve, além da seleção genética, a melhorias na nutrição, instalações e cuidados com a vaca. Entretanto, concomitantemente ao melhor desempenho produtivo, tem ocorrido uma diminuição na eficiência reprodutiva (WILTBANK, 1998; BOLAND et al., 2001). Segundo BOLAND et al. (2001), a nutrição é um dos fatores mais importantes que influenciam a fertilidade. Para ajustar as novas exigências nutricionais, foi necessário incrementar o consumo pelos animais e a adição de gordura nas dietas surgiu como alternativa. Além dos efeitos sobre a produção, a gordura tem provocado modificações na reprodução, as quais poderiam ser utilizadas para incrementar os resultados de tecnologias como a transferência de embriões.

Em trabalhos onde a gordura foi utilizada na dieta de bovinos, houve aumento do número de folículos (DE FRIES et al., 1998) ou ainda aumento do tamanho do folículo maior (DE FRIES et al., 1998; MOALLEN et al., 1999). As fontes lipídicas variam, e podem ser utilizadas gorduras insaturadas, como as provenientes de óleos vegetais (óleo de soja, farelo de arroz), ou saturadas, de origem animal (sebo, graxa amarela, farinha de peixe).

Os efeitos da nutrição sobre a resposta superovulatória e qualidade embrionária em bovinos e ovinos tem sido alvo de uma série de estudos (BOLAND et al., 2001; NEGRÃO et al., 2003). Entre os fatores nutricionais mais estudados, capazes de afetar a resposta superovulatória e qualidade embrionária, estão o aumento do consumo de alimento, as fontes de energia na dieta e o consumo de proteína (revisado por SANTOS, 2000).

O objetivo deste experimento foi estudar os efeitos de níveis de gordura (4, 6 e $8 \%$ de EE) na dieta de vacas em lactação, sobre a resposta superovulatória e qualidade embrionária.

\section{MATERIAL E MÉTODOS}

O experimento foi realizado no período de setembro de 2000 a agosto de 2001, no sistema de produção de leite (SISPEL) da Embrapa Clima Temperado, localizado em Capão do Leão, Rio Grande do Sul. Foram utilizadas dezoito vacas Jersey PO com produção média de $22,2 \pm 1,7 \mathrm{~kg}$ de leite por dia com $4,0 \%$ de gordura, com ordem de lactação variando de zero a quatro, peso médio de $420 \pm 70,6 \mathrm{~kg}$ ao parto e ciclando regularmente.

Os animais foram agrupados em bloco por data de parto e ordem de lactação, e distribuídos aleatoriamente nos tratamentos que consistiram de: um grupo controle com dieta à base de milho e farelo de soja com extrato etéreo (EE) de 4,0\% $(n=6)$ um grupo médio que recebeu a dieta controle acrescida de sebo bovino para atingir $6,0 \%$ de extrato etéreo $(n=6)$ e um grupo alto com a dieta controle acrescida de sebo bovino para atingir $8,0 \%$ de extrato etéreo $(n=6)$. $\mathrm{Na}$ tabela 1 , estão demonstrados os valores dos nutrientes das dietas fornecidos em cada tratamento.

Uma ração básica para vaca de transição foi fornecida aos animais cerca de 21 dias antes da

Tabela 1 - Composição das dietas experimentais e dos nutrientes das dietas expressa como percentagem da dieta, em base seca.

\begin{tabular}{|c|c|c|c|}
\hline Composição das dietas & Controle & Média & Alta \\
\hline Feno de alfafa & 24,7 & 24,7 & 24,7 \\
\hline Capim tifton & 1,8 & 1,8 & 1,8 \\
\hline Silagem de milho & 26,5 & 26,4 & 26,5 \\
\hline Calcário & 0,4 & 0,4 & 0,4 \\
\hline Farelo de arroz integral & 7,1 & 7,1 & 7,1 \\
\hline Farelo de soja & 12,9 & 13,5 & 14,1 \\
\hline Farinha de peixe & 1,5 & 1,5 & 1,5 \\
\hline Fosfato bicálcico & 0,8 & 0,8 & 0,8 \\
\hline Milho em grão & 23,9 & 21,2 & 18,3 \\
\hline Sal & 0,4 & 0,4 & 0,4 \\
\hline Sebo & $\mathrm{x}$ & 2,2 & 4,4 \\
\hline \multicolumn{4}{|c|}{ Composição de nutrientes das dietas } \\
\hline MO & 91,48 & 91,49 & 91,49 \\
\hline PB & 17,26 & 17,25 & 17,31 \\
\hline FDN & 32,21 & 31,81 & 31,47 \\
\hline $\mathrm{EE}$ & 3,94 & 6,03 & 8,06 \\
\hline $\mathrm{CNE}$ & 38,06 & 36,39 & 34,65 \\
\hline MM & 8,52 & 8,51 & 8,51 \\
\hline
\end{tabular}

$\mathrm{MO}=$ matéria orgânica, $\mathrm{PB}=$ proteína bruta, $\mathrm{FDN}=$ fibra detergente neutra, $\mathrm{EE}=$ extrato etéreo, $\mathrm{CNE}=$ carboidratos não estruturais, $\mathrm{MM}=$ matéria mineral Referência: BORBA et al., 2003.

Ciência Rural, v.35, n.3, mai-jun, 2005. 
data prevista para o parto e os tratamentos foram instituídos, gradualmente, 24 horas após o parto. Forneceu-se o alimento volumoso por módulo, uma vez ao dia, e o concentrado em cocho individual, três vezes ao dia. As vacas foram ordenhadas duas vezes ao dia, sendo medidas as produções individuais a cada ordenha (BORBA et al., 2003).

$\mathrm{O}$ processo de pré-sincronização estral e superovulação iniciou com o método denominado Ovsynch (PURSLEY et al., 1995) que consiste de uma aplicação inicial de $100 \mu \mathrm{g}$ de GnRH (Gestran ${ }^{\circledR}$, Tecnopec), seguida de uma dose de $25 \mathrm{mg}$ de prostaglandina (Lutalyse $\AA$, Pfizer Inc) sete dias após e, dois dias depois, de nova dose de GnRH. Seis dias depois da segunda dose de GnRH, colocou-se um implante auricular de progestágeno contendo $3 \mathrm{mg}$ de norgestomet (Crestar ${ }^{\circledR}$, Intervet) e, no dia seguinte à colocação do implante, administrou-se uma dose de benzoato de estradiol $(2,5 \mathrm{mg})$. Iniciou-se, quatro dias depois da dose de benzoato de estradiol, o programa de superovulação onde foram utilizadas $300 \mathrm{mg}$ de FSH (Pluset ${ }^{\circledR}$, Calier) em doses decrescentes, duas doses diárias com intervalo de 12 horas, por quatro dias. No terceiro dia do programa de superovulação, no período da tarde, foi retirado o implante de progesterona e, administrada nova dose de prostaglandina (Lutalyse ${ }$, Pfizer Inc). Realizou-se detecção visual dos sinais de estro e duas inseminações artificiais, uma no momento da detecção e outra doze horas após. Sete dias após a primeira inseminação, as estruturas foram recuperadas pelo método não cirúrgico (PALMA, 2001) e avaliadas quanto ao estágio de desenvolvimento e qualidade embrionária (IETS, 1999), sob lupa estereoscópica. Os animais foram superovulados duas vezes, aos 90 e aos $130 \mathrm{dpp}$ (dias pós parto). No dia da coleta das estruturas, foi realizada uma estimativa, através de palpação retal, do número de corpos lúteos presentes nos ovários. A primeira superovulação foi realizada quando os animais estavam com cerca de $90 \mathrm{dpp}$, porque este período é posterior ao período considerado crítico para vacas de alta produção, quando se encontram em balanço negativo de energia líquida. A segunda superovulação foi realizada aos $130 \mathrm{dpp}$, devido ao intervalo necessário para sincronizar e superovular os animais antes de uma nova coleta.

Foi realizada análise dos dados de temperatura ambiental média diária durante os tratamentos superovulatórios. Para as análises da concentração de progesterona, foram coletadas amostras de sangue no dia da colocação do implante, durante o tratamento de superovulação, nos dois dias anteriores à coleta e no dia da coleta de embriões. As amostras foram analisadas por rádio imunoensaio (Coat-a-Cout ${ }^{\circledR}$ Progesterone) e a leitura da radioatividade remanescente foi realizada em contador gama.

No experimento, foi utilizado o delineamento em blocos casualizados composto (6 blocos), tomando como base a data de parto e a ordem de lactação. Os animais foram utilizados como unidade experimental para avaliação dos dados de recuperação embrionária e progesterona, e os embriões foram considerados como unidade experimental na análise de qualidade e desenvolvimento embrionário. Os dados foram tratados por Análise da Variância para dados repetidos usando o procedimento PROC MIXED do SAS ${ }^{\circledR}$ e utilizando a função auto regressiva (AR) como opção de covariância. $\mathrm{O}$ modelo matemático incluiu tratamento, bloco, período, animal dentro de tratamento e erro experimental. Os resultados são apresentados como média dos quadrados mínimos e erro padrão da média. As diferenças médias foram consideradas significativas com probabilidades $<0,05$ e tendência com probabilidades entre $>0,05 \mathrm{e}<0,10$.

\section{RESULTADOS E DISCUSSÃO}

As dietas testadas não alteraram a eficácia da coleta em relação ao número de estruturas recuperadas, o número de corpos lúteos e as concentrações séricas de progesterona no dia da colocação do implante, durante a superovulação e no dia da coleta, conforme demonstra a tabela 2 . Entretanto, houve uma tendência de efeito do tratamento $(\mathrm{P}=0,09)$ onde, o grupo de vacas que recebeu o tratamento alto ( $8 \%$ de EE) apresentou maior número de estruturas em relação ao que recebeu tratamento médio $(6 \%$ de $\mathrm{EE})(\mathrm{P}=0,03)$, sendo $6,2 \pm 1,5 \mathrm{e}$ $1,1 \pm 1,6$, respectivamente.

Houve tendência de efeito do período de superovulação $(\mathrm{P}=0,07)$ para o número de estruturas recuperadas com média dos quadrados mínimos e erro padrão da média (MQM \pm EPM) de 4,3 $\pm 0,9$ vs 2,9 $\pm 0,9$ nas superovulações realizadas aos 90 e 130dpp, respectivamente. Adicionalmente, foi encontrada tendência para efeito do período $(\mathrm{P}=0,06)$ sobre o número de corpos lúteos, $11,0 \pm 0,9$ e $8,9 \pm 0,9$ nas superovulações realizadas aos 90 e $130 \mathrm{dpp}$, respectivamente.

Esperava-se que, com a utilização da gordura, ocorresse aumento do número de folículos recrutados, frente ao tratamento com $\mathrm{FSH}$, e que um maior número de estruturas fossem recuperadas, incrementando os resultados da técnica nos animais responsivos. A adição de gordura na dieta tem 
Tabela 2 - Efeito da adição de gordura na dieta sobre a eficiência da coleta em relação ao número de estruturas, corpos lúteos e progesterona plasmática (ng/ml) (MQM \pm EPM).

\begin{tabular}{|c|c|c|c|c|c|c|}
\hline & \multicolumn{3}{|c|}{ Tratamento } & \multicolumn{3}{|c|}{$\mathrm{P}<\mathrm{F}$} \\
\hline & Controle & Médio & Alto & Tratamento & Período & Tratamento*Período \\
\hline Estruturas & $3,5 \pm 1,5$ & $1,1 \pm 1,6$ & $6,2 \pm 1,5$ & 0,09 & 0,07 & 0,53 \\
\hline Jorpos lúteos & $9,6 \pm 1,4$ & $10,4 \pm 1,1$ & $9,8 \pm 1,1$ & 0,88 & 0,06 & 0,12 \\
\hline Jrogesterona Implante* & $3,0 \pm 1,6$ & $5,5 \pm 1,2$ & $3,9 \pm 1,2$ & 0,43 & 0,79 & 0,19 \\
\hline ?rogesterona $\mathrm{SOV}^{* *}$ & $4,4 \pm 0,8$ & $3,5 \pm 0,7$ & $2,8 \pm 0,7$ & 0,35 & 0,22 & 0,87 \\
\hline Progesterona coleta $* * *$ & $22,3 \pm 3,9$ & $19,1 \pm 4,1$ & $16,6 \pm 3,5$ & 0,56 & 0,21 & 0,88 \\
\hline
\end{tabular}

*Progesterona plasmática no dia da colocação do implante de progesterona, **Progesterona média durante o tratamento de superovulação e, k** média da progesterona plasmática obtida a partir de amostras coletadas nos dois dias anteriores à coleta e no dia da coleta das estruturas. _etras diferentes na mesma linha diferem estatisticamente. MQM= média dos quadrados mínimos, EPM= erro padrão da média

provocado aumento do número de folículos de tamanho médio ou ainda aumento do tamanho do folículo dominante (DE FRIES et al., 1998; MOALLEM et al., 1999). Entretanto, os resultados deste experimento confirmaram o encontrado por THOMAS \& WILLIAMS (1996), quando utilizaram dietas com óleo de soja e sebo, que não encontraram diferença no número de ovulações após tratamento de superovulação. Embora não tenha sido diferente para os tratamentos, o número médio estimado de corpos lúteos deste experimento $(9,6 \pm 1,4 ; 10,4 \pm 1,1$ e $9,8 \pm 1,1$ para controle, médio e alto, respectivamente) foi semelhante ao encontrado por THOMAS \& WILLIAMS (1996) pela contagem após ovariectomia,

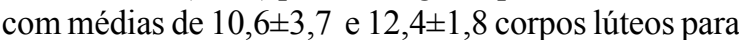
os tratamentos com sebo e óleo de soja, respectivamente.

MIHM \& AUSTIN (2002) sugeriram um mecanismo pelo qual uma dieta suplementada com gordura provocaria o incremento do número de folículos recrutados. Segundo os autores, a adição de gordura aumentaria o nível plasmático e folicular de HDL- colesterol (colesterol ligado à lipoproteína de alta densidade), o qual, uma vez livre nas células luteais da granulosa, estimularia a produção de IGF-I (fator de crescimento semelhante à insulina) e outros fatores de crescimento. ARMSTRONG et al. (2001) descreveram que a composição da dieta altera a expressão de RNAm para a tradução de IGF ovariano, suportando a hipótese da regulação nutricional sobre o crescimento folicular. Também observaram que novilhas que receberam dietas com alta energia apresentaram uma diminuição na expressão de RNAm para codificação de proteínas de ligação à IGF em folículos pequenos, o que disponibilizaria mais IGF livre nestes folículos. Conseqüentemente, aumentaria a sensibilidade ao FSH e aumentaria a taxa de crescimento folicular.

Quanto à qualidade, o número de embriões (graus 1 a 3; tabela 3 ) foi diferente entre os tratamentos $(\mathrm{P}=0,03)$. Os animais que receberam o tratamento médio produziram menor número de embriões viáveis $(0,3 \pm 1,3)$ em relação ao tratamento alto $(5,3 \pm 1,2)$, entretanto, não diferiram do tratamento controle $(3,9 \pm 1,1)$

Neste experimento, 55,5\% dos animais produziram estruturas viáveis e $44,4 \%$ não produziram estruturas, independente dos tratamentos testados. A percentagem de animais que produziu estruturas viáveis é um pouco inferior ao encontrado por CABODEVILA \& TORQUATI (2001), os quais observaram que somente cerca de $68 \%$ de 1263

Tabela 3 - Efeito da adição de gordura na dieta sobre o rendimento e qualidade embrionária (MQM EPM).

\begin{tabular}{lcccccc}
\hline & \multicolumn{2}{c}{ Médias dos quadrados mínimos para tratamento } & & $\mathrm{P}<\mathrm{F}$ \\
\cline { 2 - 7 } & Controle & Médio & Alto & Tratamento & Período & Tratamento*Período \\
\hline Embriões $^{1}$ & $3,9 \pm 1,1^{\text {ab }}$ & $0,3 \pm 1,3^{\mathrm{b}}$ & $5,3 \pm 1,2^{\mathrm{a}}$ & 0,03 & 0,38 & 0,13 \\
Graus 1 e 2 $^{2}$ & $3,1 \pm 0,9$ & $0,4 \pm 1,1$ & $2,1 \pm 1,0$ & 0,18 & 0,74 & 0,37 \\
Grau 3 $^{3}$ & $0,2 \pm 0,4^{\mathrm{b}}$ & $0,0 \pm 0,5^{\mathrm{b}}$ & $2,9 \pm 0,4^{\mathrm{a}}$ & 0,001 & 0,61 & 0,16 \\
Grau 4 $^{4}$ & $0,5 \pm 0,5$ & $0,9 \pm 0,4$ & $1,3 \pm 0,4$ & 0,47 & 0,001 & 0,60 \\
\hline
\end{tabular}

a,b Letras diferentes na mesma linha diferem estatisticamente. ${ }^{1}$ Embriões grau 1,2 e $3 ;{ }^{2}$ Grau $1=$ excelente; ${ }^{2}$ grau $2=$ bom; ${ }^{3}$ grau $3=$ regular; ${ }^{4}$ grau=4 degenerado + não fecundados. MQM= média dos quadrados mínimos, EPM= erro padrão da média 
doadoras produziram embriões transferíveis. Entretanto, vários fatores podem interferir no sucesso da Tecnologia de Embriões (CABODEVILA \& TORQUATI, 2001; REICHENBACH, 2003) e um fator importante deste experimento é que foram utilizados animais em lactação que possuem exigências nutricionais adicionais.

O número de embriões com graus 1 e 2 (Tabela 3) não foi diferente para os tratamentos $(\mathrm{P}=0,18)$, entretanto, os animais que receberam dieta com alta gordura produziram maior número de embriões de grau $3(2,9 \pm 0,4)(\mathrm{P}<0,01)$ em relação aos grupos que receberam dietas controle $(0,2 \pm 0,4)$ e médio $(0,0 \pm 0,5)$. Não houve diferença significativa em relação ao número de embriões de grau 4 (degenerados e não fecundados; tabela 3) para os tratamentos $(\mathrm{P}=0,47)$. Entretanto, quando analisado o efeito do período da superovulação, os animais produziram maior número de estruturas de grau 4 aos $90 \mathrm{dpp}$, em relação aos que foram coletados $130 \mathrm{dpp}(\mathrm{P}=0,001)$. Os animais produziram em média $1,6 \pm 0,3$ embriões de grau 4 aos 90 e $0,2 \pm 0,3$ aos $130 \mathrm{dpp}(\mathrm{P}=0,0015)$. O aumento no número de embriões de grau 4 poderia estar relacionado ao status energético do animal. Se o animal ainda não estivesse em balanço positivo de energia líquida, poderia estar debilitado e, assim, as funções reprodutivas estariam sendo prejudicadas, produzindo aumento de embriões de qualidade inferior neste período. Adicionalmente, os ovócitos levam cerca de 80 a 100 dias para se desenvolver de folículo primordial até se tornar ovulatório. Desta forma, as estruturas coletadas na superovulação aos 90dpp são originárias de um período em que os animais se encontravam em fase inicial de lactação, e as exigências nutricionais eram deficientes, podendo interferir na qualidade dos ovócitos e conseqüentemente, dos embriões produzidos.

O efeito do período de superovulação para embriões de grau 4 não foi correlacionado significativamente $(\mathrm{r}=0,30 ; \mathrm{P}=0,08)$ com as temperaturas médias durante o tratamento de superovulação aos 90 e $130 \mathrm{dpp}$, apesar das temperaturas médias terem se apresentado mais altas $(\mathrm{P}<0,01)$ durante os tratamentos de superovulação realizados aos $90 \mathrm{dpp}\left(22,5 \pm 0,45^{\circ} \mathrm{C}\right)$ em relação aos $130 \mathrm{dpp}\left(19,7 \pm 0,45^{\circ} \mathrm{C}\right), \mathrm{r}=-0,62$ $(\mathrm{P}=<0,01)$. No entanto, estas diferenças de temperatura não seriam suficientes para influenciar a qualidade embrionária, uma vez que estão próximas do considerado como temperatura de conforto para os animais.

Vários trabalhos que utilizaram gordura na dieta observaram aumento do tamanho do folículo maior (DE FRIES et al., 1998; MOALLEN et al., 1999). Este fator poderia ser considerado quando se avalia o aumento do número de estruturas de grau 3. Se a gordura aumentou o tamanho destes folículos e eles vieram a ovular um ovócito de qualidade inferior, isto poderia contribuir, também, com a produção de um embrião de qualidade inferior. Adicionalmente, se a gordura é capaz de alterar a quantidade de hormônios produzida, também pode provocar modificações no ambiente tubárico e uterino, que por sua vez, podem não estar adequados tanto para a fecundação como para o desenvolvimento embrionário.

As concentrações séricas de progesterona (Tabela 2) não foram diferentes entre os tratamentos testados no dia da colocação do implante $(\mathrm{P}=0,43)$, durante o programa de superovulação $(\mathrm{P}=0,35)$, ou na média entre os dois dias que antecederam a coleta $\mathrm{e}$ no dia da coleta $(\mathrm{P}=0,56)$. A progesterona plasmática nos dias 5 e 7 após a inseminação artificial tem sido positivamente correlacionada com embriões viáveis, ovócitos fecundados e total de ovócitos recuperados (CHAGAS \& SILVA et al., 2002). Neste experimento, não foram encontradas correlações significativas das concentrações plasmáticas de progesterona com o número de estruturas coletadas $(\mathrm{r}=0,21 ; \mathrm{P}=0,23)$, o número de embriões de graus 1 e $2(\mathrm{r}=0,07 ; \mathrm{P}=0,70)$, o número de embriões de grau $3(\mathrm{r}=0,13 ; \mathrm{P}=0,47)$, o número de embriões de grau $4(\mathrm{r}=0,32 ; \mathrm{P}=0,07)$, e o número de não fecundados ( $\mathrm{r}=-0,15 ; \mathrm{P}=0,39)$.

$\mathrm{O}$ consumo de matéria seca (BORBA et al., 2003) não foi alterado pelos tratamentos. Também MALAFAIA et al. (1996) não encontraram diferenças no consumo de matéria seca, quando utilizaram dietas com 4,7 e $10 \%$ de sebo bovino. Isto pode ser devido ao fato de que a fonte de gordura utilizada para incrementar o extrato etéreo foi o sebo, gordura de origem animal que tem pouca ação sobre o metabolismo ruminal (SANTOS \& AMSTALDEN, 1998). Segundo SANTOS \& AMSTALDEN (1998), o consumo de matéria seca tende a diminuir nas primeiras semanas pós-parto, quando os animais recebem gordura na dieta, oque foi observado por FERGUSON et al. (1990), os quais, ainda obtiveram maior taxa de concepção.

Os resultados deste experimento demonstraram que a adição de gordura em nível de $8 \%$ de extrato etéreo é prejudicial à qualidade embrionária, pois os animais que receberam esta dieta apresentaram maior número de embriões de qualidade inferior. Entretanto, novos experimentos são necessários para elucidar os possíveis mecanismos envolvidos com os efeitos da gordura sobre a resposta superovulatória.

Ciência Rural, v.35, n.3, mai-jun, 2005. 


\section{CONCLUSÃO}

A adição de diferentes níveis de gordura não melhora a resposta superovulatória e a qualidade embrionária.

\section{AGRADECIMENTOS}

Os autores agradecem a Capes pelo suporte financeiro da aluna Coscioni, A.C., ao Programa de Pós graduação em Zootecnia da UFPel e à Embrapa Clima Temperado, pelo aporte financeiro para realização do experimento.

\section{REFERÊNCIAS BIBLIOGRÁFICAS}

ARMSTRONG, D.G. et al. Effect of dietary energy and protein on bovine follicular dynamics and embryo production in vitro: associations with the ovarian insulin-like growth factor system. Biology of Reproduction, v.64, p.1624-1632, 2001 .

BOLAND, M.P. et al. Effect of nutrition on endocrine parameters, ovarian physiology, and oocyte and embryo development. Theriogenology, v.55, p.1323-1340, 2001.

BORBA, L.R.O. et al. Efeitos da adição de níveis crescentes de gordura na dieta de vacas leiteiras de alta produção. Agrociência, 2003 (no prelo).

CABODEVILA, J.; TORQUATI, S. Superovulación de hembras bovinas. In: PALMA, G.A. Biotecnología de la Reproducción. Balcarce, Argentina : Ediciones, Instituto Nacional de Tecnología Agropecuaria, 2001. Cap.VI, p.79108

CHAGAS E SILVA, J. et al. Embryo yield and plasma progesterone profiles in superovulated dairy cows and heifers. Animal Reproduction Science, v.69, n.1-2, p.1-8, 2002 .

DE FRIES, C.A. et al. Fat supplementation influences postpartum reproductive performance in Brahman cows. Journal of Animal Science, v.76, p.864-870, 1998.

FERGUSON, J.D. et al. Effects of hard fats on in vitro and in vivo rumen fermentation milk production, and reproduction in dairy cows. Journal of Dairy Science, v.73, p.28642879, 1990.

IETS (International Embryo Transfer Society). Manual da Sociedade Internacional de Transferência de Embriões. Illinois, USA : Savoy, 1999. 180p.

LOPEZ, S.E. et al. Supplementation with different fat sources on intake, milk yield and feed efficiency of cows in early lactation. In: WORLD CONFERENCE OF ANIMAL PRODUCTION, 9., AND REUNIÃO DA ASSOCIAÇÃO LATINO AMERICANA DE PRODUÇÃO ANIMAL, 18. 2003, Porto Alegre. Anais... Viçosa, MG : SBZ, 2003. 1 CD.
MALAFAIA, P.A.M. et al. Sebo bovino em rações para vacas em lactação. 1. Consumo de nutrientes, produção e composição do leite. Revista da Sociedade Brasileira de Zootecnia, v.25, n.1, p.153-163. 1996.

MIHM, M.; AUSTIN, E.J. The final stages of dominant follicle selection in cattle. Domestic Animal Endocrinology, v.23, p. 155-166, 2002 .

MOALLEM, U. et al. Effect of calcium soaps of fatty acids and administration of somatotropin on milk production, preovulatory follicular development, and plasma and follicular fluid lipid composition in high yielding cows. Journal of Dairy Science, v.82, p.2358-2368, 1999.

NEGRÃO, S.L. et al. Efeito dos níveis de energia da dieta na taxa de recuperação de embriões e nas concentrações plasmáticas de progesterona, estradiol e glicose em vacas doadoras de embriões. Acta Scientiae Veterinariae, v.31, Supl, p.502, 2003.

NÖRNBERG, J.L. et al. Valor do farelo de arroz integral como fonte de gordura na dieta de vacas Jersey na fase inicial de lactação. Revista Brasileira de Zootecnia, Viçosa-MG, (no prelo) 2004.

PALMA, G.A. Biotecnología de la reproducción. Balcarce: Instituto Nacional de Tecnología Agropecuaria (INTA), 2001. 701p.

PURSLEY, J.R. et al. Synchronization of ovulation in dairy cows using PGF2a and GnRH. Theriogenology, v.44, p.915923, 1995.

REICHENBACH, H.-D. Embryo transfer and cryopreservation in cattle: practical considerations. Acta Scientiae Veterinariae, v.31, Supl, p.28-50, 2003.

SANTOS, J.E.P.; AMSTALDEN, M. Effects of nutrition on bovine reproduction. Arquivos da Faculdade de Veterinária UFRGS, Porto Alegre, v.26, n.1, p.19-89, 1998.

SANTOS, J.E.P. Importância da alimentação na reprodução da fêmea bovina. In: WORKSHOP SOBRE REPRODUÇÃO ANIMAL, 1., 2000, Pelotas, RS. Anais... Pelotas : Embrapa Clima Temperado, 2000. V.1, p.7-82.

THOMAS, M.G.; WILliAMS, G.L. Metabolic hormone secretion and FSH-induced superovulatory responses of beef heifers fed dietary fat supplements containing predominantly saturated or polyunsaturated fatty acids. Theriogenology, v.45, p.451-458, 1996 .

WEIGEL, A.K. Melhoramento genético de vacas leiteiras com enfoque na longevidade e eficiência reprodutiva. In: CURSO "NOVOS ENFOQUES NA PRODUÇÃO E REPRODUÇÃO DE BOVINOS” 5., 2001, Uberlândia, MG. Anais... Botucatu, SP : Conapec Jr, 2001. p.1-5.

WILTBANK, M.C. Improving reproductive efficiency in high producing dairy cattle. In: CURSO "NOVOS ENFOQUES NA PRODUÇÃO E REPRODUÇÃO DE BOVINOS”, 2., 1998, Uberlândia, MG. Anais... Botucatu, SP : Conapec Jr, 1998. p.70-89. 